\title{
Possibility of the drag reduction effect application to reduce the cost of transport energy in water systems
}

\author{
Zbigniew Matras ${ }^{1}$, and Bartosz Kopiczak ${ }^{1, *}$ \\ ${ }^{1}$ Institute of Thermal and Process Engineering, Cracow University of Technology, Cracow, Poland
}

\begin{abstract}
The main aim of this paper is to present possible drag reduction effect application to reduce the energy costs in water transport systems. Results obtained in laboratory scale experiment present possibility to enhance the drag reduction effect in pipe flow by simultaneous addition to the transported water small amount of high molecular polymers and surfactants. The hypothetic mechanism of drag reduction by polymer-micellar aggregates is presented. Qualitative analysis of polymer-micellar additives influence on shape and character of flow resistance curves is performed. Complex polymer-micellar solution flow resistance curves are compared with appropriate single additive polymer or surfactant one.
\end{abstract}

\section{Introduction}

The most commonly used drag reduction (DR) agents in scientific research as well as in engineering applications are high molecular weight polymers and surfactants. Adding even a small amount of these substances to the transported solvent induces significant and extremely advantageous, from the energetic point of view, reductions of pressure losses caused by fluid internal friction in the turbulent range of flow. This results in a significant increase in the flow rate without the necessity of increasing the power demand, or vice versa - to reduce the power demand while maintaining a constant flow rate [1-7]. Therefore, it provides large potential possibilities for application of this effect in different industry branches, particularly in oil industry $[3,8]$, or in heating [9], fire-fighting [10], transport of slurries, sludge and brines $[2,11]$, while increasing the efficiency of sewerage and anti-flood systems in time of heavy rainfall [12]. The drag reduction effect caused by polymers was utilized in designing an industrial installation for crude oil transportation in Norway, USA and India $[2,3,8]$.

The new internal structure, which occurs when special additives are introduced into the solution, was perceived to be the cause of the drag reduction effect.

Adding a small amount of a polymer to the water causes a change of the internal structure of the solution creating so called macromolecules. Due to the characteristic chain structure of polymers in solutions, macromolecules form different conformations.

The drag reduction effect caused by small amounts of surfactant addition is particularly interesting from a cognitive point of view, as well as its utilitarian character [1, 2, 13-19]. Surfactants added to the water solvent create a low concentrated solution. When the surfactant concentration exceeds a certain critical level of concentration, individual surfactant molecules start to organize into new structures called micelles. The drag reduction effect in the flow of such a micellar solution takes place only when cylindrical/threadlike micelles are present in the solution. Reformation of spherical micelles into threadlike micelles is usually induced by adding a small amount of electrolytes (e.g. salts or alcohols) into the surfactant solution [14-17].

At no motion condition, when the fluid is at rest, the above mentioned structures are chaotic. Only during fluid flow shearing, both macromolecules and micelles start to arrange in a characteristic orientation, in accordance with the principle of minimum resistance. Solution concentrations are very small. In case of high molecular polymers it is order of magnitude of several or several dozen of ppm, and for surfactants it is order of tenths of $\mathrm{mM} / \mathrm{dm} 3$.

The effect of drag reduction of flow by high molecular polymers or surfactants has also some limitations resulting from the properties of additives. The main disadvantage of high molecular polymers is their susceptibility to mechanical and thermal degradation. In case of surfactant solutions it is a certain critical Reynolds number. When exceeded, micelles orientation is lost, and the drag reduction effect collapses. The number depends not only on the pipe's diameter, but also on the solution concentration.

An important distinguishing factor for the phenomenon of drag reduction caused by surfactant additives comparing to drag reduction effect induced by high molecular polymers is the reversibility of solution degradation $[1,15]$. In case where the value of $\mathrm{Re}$ number falls below critical Re value, the drag reduction effect occurs again. Moreover, the drag reduction effect in surfactant solution is not weakened in time of longterm liquid pumping comparing to the polymer solution.

\footnotetext{
*Corresponding author: bartosz.kopiczak@mech.pk.edu.pl
} 
This is a great advantage for the transport of liquids over long distances.

The new and poorly recognized effect is phenomenon of fluid flow drag reduction by co-addition into the solvent both the high molecular polymer and the surfactant with salt. Mainly internal structure formation and chemical reaction process in polymer-micellar solutions are highlighted in the few published works related with this subject [18-20]. First attempts of drag reduction effect experimental examination had been performed, confirming that simultaneous introduction into the solvent polymer and surfactant additives combine positive features of their purely polymer and micellar analogues providing additional extension of drag reduction zones $[15,16,21]$. The pipeline drag reduction measurements reveal a considerable synergistic effect, that is, the mixed polymer-surfactant aggregates gives a significantly higher drag reduction comparing to their pure polymer or pure surfactant equivalents.

The aim of the work is experimental confirmation of the thesis that co-addition to the solvent both surfactants and high molecular polymer causes intensification of drag reduction effect and significant extension of drag reduction zones, comparing to the drag reduction effect obtained by application of pure polymer or pure surfactant additives. Possible drag reduction effect application to reduce the costs of transport energy in water transport systems will be proposed.

\section{Laboratory set-up and DR additives}

Measurements were performed using modern capillary-pipe rheometer, designed and constructed in the Division of Fluid Mechanics laboratory at the Cracow University of Technology [22]. Additionally rheological characteristics of the analysed solution have been assigned by the use of the Anton Paar rotational rheometer MRC 301 with the plate-cone measuring system. The following drag reduction agents were used for experimental analysis: the non-ionic polymer poly(ethylene oxide) $-[\mathrm{CH} 2 \mathrm{CH} 2 \mathrm{O}] \mathrm{n}$ (PEO) with high molecular weight $\mathrm{Mv}=8.106 \mathrm{~g} / \mathrm{mol}$ and the cationic surfactant - cetyltrimetyl ammonium bromide [CH3(CH2)and5N(CH3)3]+Br $(\mathrm{CTAB})$.

In order to lower the $\mathrm{CMC}$ value, salt sodium salicylate $\mathrm{C} 7 \mathrm{H} 5 \mathrm{NaO} 3$ (NaSal) was used. Distilled water was used as the solvent.

Adiabatic steady flow of homogenous solutions were examined in 4 different straight pipes with diameters between $10[\mathrm{~mm}]$ and $21[\mathrm{~mm}]$, in temperature equal to $25^{\circ} \mathrm{C}$.

\section{Experimental analysis and discussion}

In order to identify rheological characteristics of analysed solution, flow curves have been drawn for each of them in form of relationship described by the equation (1):

$$
\tau=\mathrm{f}(\dot{\gamma})
$$

where

$\tau \quad-$ the shear stress,

$\dot{\gamma} \quad-$ the shear rate.

Interpretation of experimental results presented in form of flow curves indicates, that analysed solutions can be successfully approximated with the Ostwald de Waele power-law fluid model. Representative rheological characteristics are illustrated in figure 1 .

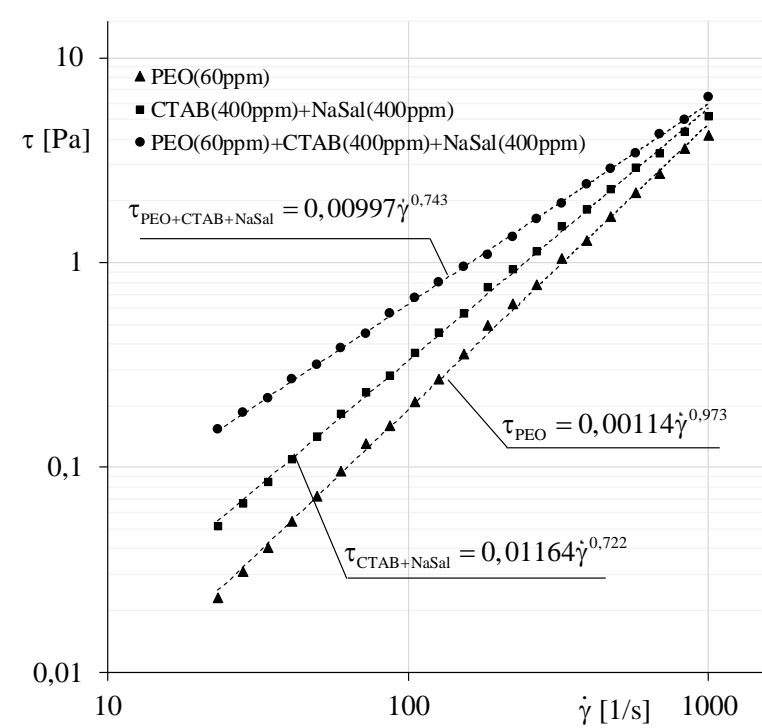

Fig. 1. Representative flow curves of polymer, surfactant and polymer-surfactant solutions.

In order to perform a better interpretation of the effect of the simultaneous addition of the polymer, the surfactant and salt, comparing to the adequate addition of pure polymer or pure surfactant, measurement data was presented in the modified system of "pseudorheostable" numbers [Re $\left.\mathrm{Re}_{\mathrm{M}}, \mathrm{c}_{\mathrm{fM}}\right]$, and described by formulas (2) and (3) [7]:

$$
\begin{gathered}
c_{\mathrm{fM}}=\frac{\frac{\mathrm{D} \Delta \mathrm{p}}{4 \mathrm{~L}}}{\frac{\rho \mathrm{u}_{\mathrm{m}}^{2}}{2}}\left[\frac{2(\mathrm{n}+1)}{3 \mathrm{n}+1}\right]^{2,5} \\
\operatorname{Re}_{\mathrm{M}}=\frac{\mathrm{D}^{\mathrm{n} \mathrm{u}_{\mathrm{m}}^{2-\mathrm{n}} \rho}}{\mathrm{K}\left(\frac{3 \mathrm{n}+1}{4 \mathrm{n}}\right)^{\mathrm{n}} 8^{\mathrm{n}-1}}\left[\frac{2(\mathrm{n}+1)}{3 \mathrm{n}+1}\right]^{-2,5}
\end{gathered}
$$

where:

$$
\begin{aligned}
& \mathrm{D} \quad-\text { the pipe diameter, } \\
& \rho \quad-\text { the fluid density, } \\
& \mathrm{n}, \mathrm{K} \quad-\text { the flow index and the consistency } \\
&
\end{aligned}
$$

The flow resistance curves of rheostable (purely viscous) non-Newtonian fluids in such defined dimensionless numbers system are transformed to a single curve - in the whole range of the modified Reynolds number (3) - identical to the classical Newtonian curve described in the laminar range by Fanning equation and in the turbulent flow by Blasius 
formula. The choice of such a coordinate system was dictated additionally by the fact that it facilitates the identification and description of the characteristic drag reduction flow zones. In this modified system of pseudorheostable numbers $\left[\mathrm{Re}_{\mathrm{M}}, \mathrm{c}_{\mathrm{fM}}\right.$ ] each deviation of the experimental flow resistance curve which indicates abnormal drag reduction from pseudorheostable Blasius curve, allows the identification of specific additives' influence (polymers or/and surfactants with salt) on a range of analyses of the drag reduction effect.

Representative flow resistance curves of the analysed polymer-micellar solution and the corresponding purely polymer solution and purely micellar solution are presented in figure 2 .

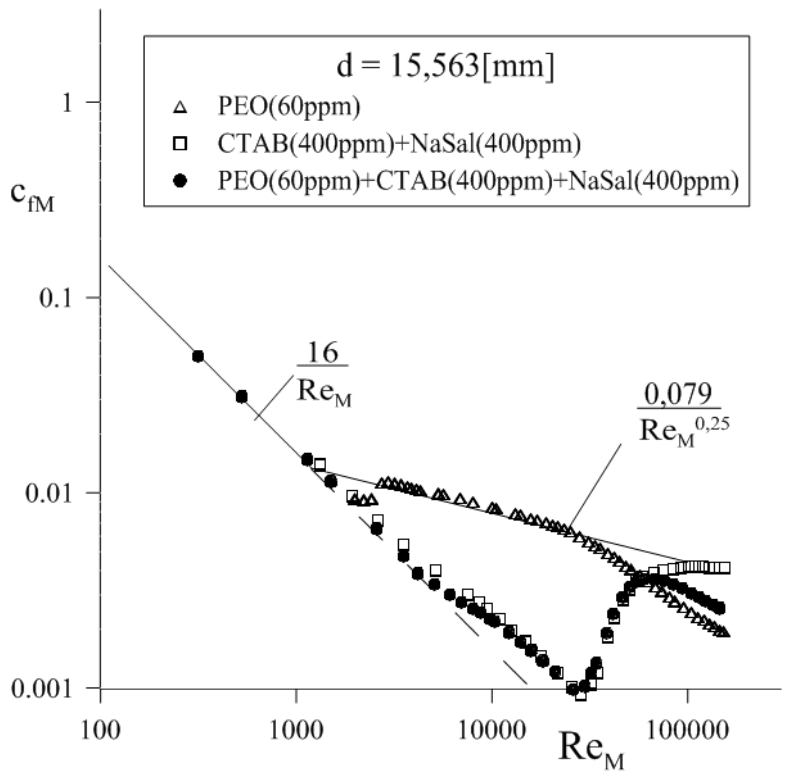

Fig. 2. Representative experimental flow resistance curves of polymer, surfactant and polymer-surfactant solutions.

The analysis of presented flow resistance curves indicates that in the laminar range of flow simultaneous addition of polymer and surfactant to the solvent causes inconsiderable increase of flow resistance and significant extension of the stable transitional zone of flow. The existence of the third significantly extended reduction zone is also observed, in which the viscoelastic properties of the solution are the dominant factor. It is particularly well illustrated by figure 3 .

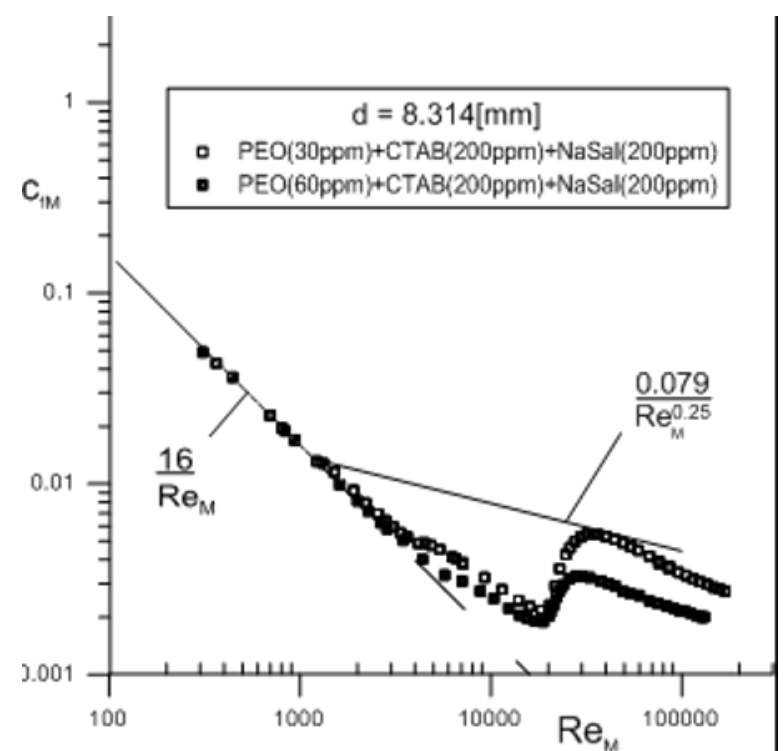

Fig. 3. Comparison of the flow resistance curves of polymersurfactant water solutions for different polymer concentration.

In comparison to a purely micellar solution, the collapse of drag reduction is normally observed in this region. It should also be noted that addition to the micellar solution of even small amounts of high molecular weight polymer (about 10-100[ppm]) causes a reduction of the non-Newtonian properties of the solution.

The results of drag reduction measurements analysis indicate the effect of pipe diameter influence on drag reduction efficiency. Increasing the pipe diameter $d$ results in clear extension of the stable transitional zone towards higher values of the Reynolds number. Moreover, decreasing the pipe diameter value $d$ results in an increase of the drag reduction effect in the third additional turbulent range of flow - figure 4.

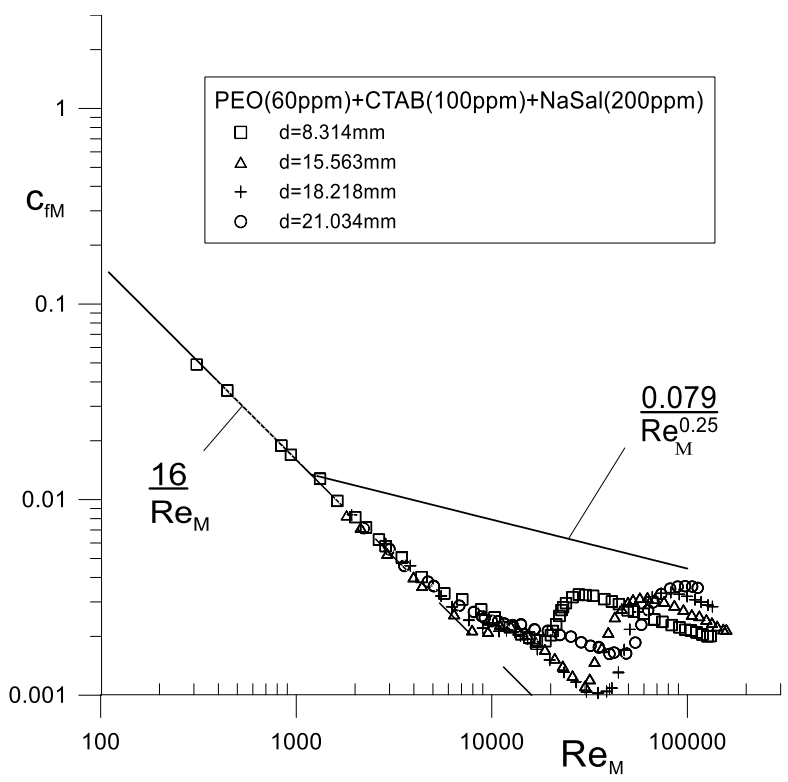

Fig. 4. Representative experimental flow resistance curves of polymer, surfactant and polymer-surfactant solutions. 
Simultaneous introduction of small amounts of polymer and surfactant additives to the solvent provides initiation of micellarization process at much lower concentration, comparing critical micelles concentrations (CMC). This concentration is called the critical aggregation concentration (CAC) $[15,20]$.

In case of an ionic surfactant mixed with a counter charged polyelectrolyte only a small part of the polyelectrolyte is adsorbed by the surfaces of the micelles. Furthermore, CAC has a lower order of magnitude than the original $\mathrm{CMC}$ due to the facts electrostatic or hydrophobic interaction occurs between the polymer and the surface of the micelles. Polymer can trigger a certain amount of concentrated counter-ions, as they combine with the micelles.

In the initial state, there is a long polymer chain with a great number of concentrated counter-ions. The final state of the mixture has single threadlike micelles with a part of polymer macromolecule chain coiled around rigid micelles. According to $[15,20,21]$, these molecules form the so-called aggregates.

Such newly created polymer-micellar solution can be characterized by a lower susceptibility to mechanical degradation during flow or its degradation can be almost invisible. Schematically illustrated aggregates (figure 5) subjected to the shear stress take orientations consistent with the aforementioned principle of minimum resistance. With increasing value of the Reynolds number, internal friction forces recoil, stretch and extend the aggregates leading to the laminarization of the initial phase of the turbulent flow.

Therefore, it may be hypothesized that the rigid rodlike micelles, which create the core of the aggregates, are responsible for reducing the flow resistance in the extended transitional zone between the laminar and turbulent flow.

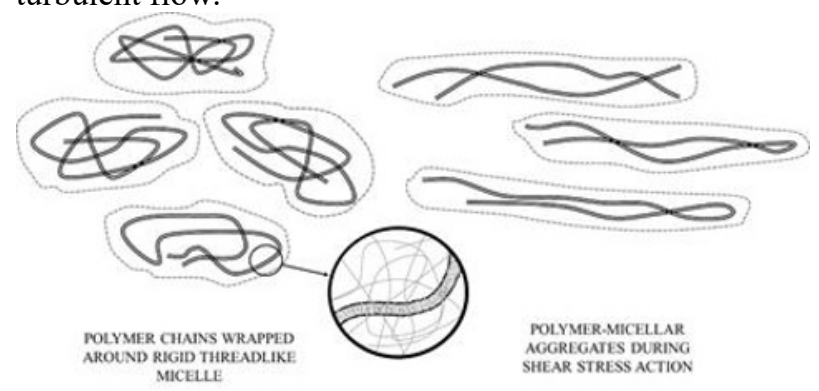

Fig. 5. Polymer-micellar aggregates.

The aggregates and micelles are responsible for transmission of internal friction in the liquid. The value of the critical Reynolds number for which the transition to the turbulent zone is observed is greater for polymermicellar solutions. This means that the stable transition zone is extended. The reason for such behaviour can be the partial disintegration of aggregates to original forms, i.e. micelles (formed from the surfactant) and macromolecules (formed from the polymer) due to a significant increase of the shear rate. From this moment, both micelles and macromolecules interact separately on the transported solution causing a further drag reduction effect. Passing further in the turbulent range of flow micelles lose their orientation and no longer have a major impact on the drag reduction. A key role is played in this zone by the polymer. Not having undergone an earlier degradation, the polymer macromolecules still cause the low reduction.

In drag reduction caused by the use of polymersurfactant solution, one cannot talk about the so-called collapse of the drag reduction. It occurs permanently over a wide range of Reynolds numbers. In the turbulent zone polymer macromolecules undergo a certain mechanical degradation. Decreasing the shear rate leads to the reconstruction of the solution's internal structure. As a result of electrostatic interaction, the recreated micelles are combined with the polymer chains by coiling around them. These chains are much shorter and such newly created aggregates do not have the same rheological properties as the original ones. This results in a slight increase of the flow resistance in comparison with a freshly prepared solution.

\section{Conclusions}

The experimental analysis of the influence of simultaneous addition of polymer and surfactant additives to a water solvent indicates that in the laminar range of flow the simultaneous addition of a polymer and a surfactant to the solvent causes an inconsiderable increase of the flow resistance comparing to the pure solvent's flow resistance. A significant extension of the stable transitional zone of flow has been observed, however. The analysis of experimental flow resistance curves (fig.2-4) allows to observe the existence of the third significantly extended reduction zone in the turbulent range of flow. In this zone the viscoelastic properties of the solution are the dominant factor. It is found that in this zone the drag reduction is influenced by the polymer concentration in the solvent. The higher concentration of polymer in the solution is applied, the greater drag reduction in the flow is obtained.

Simultaneous addition of a polymer and a surfactant with salt significantly reduces the mechanical degradation of the polymer-micellar solution's internal structure.

The comparison analysis indicates that polymermicellar solutions combine and intensify the positive features of their pure polymer and micellar equivalents. Moreover, they provide efficient reduction of flow resistance in a wider range of the Reynolds number.

Significant increase in the flow rate without the necessity of increasing the power demand, or vice versa - to reduce the power demand while maintaining a constant flow rate, which can be obtained by co-addition of polymer and surfactant to water solvent provides large potential possibilities for application of this effect in different industry branches. There are already know applications of pure surfactant solution in heating [9]. As it is expected, the drag reduction effect is accompanied by the effect of reduction of heat transfer abilities. It is anticipated from the analogues relationship between heat transport and momentum transport. This fact can be beneficial to some particular practice use of drag reduction solutions. In case of central heating systems 
and cooling systems it can greatly reduce the costs of heat insulation in pipelines - especial when water is transported at long distances. Mechanical and thermal degradation of polymer will be beneficial in places where heat exchangers are expected to supply or remove heat.

It must be pointed out, that currently, parallel to the experimental studies on the drag reduction effect, research on a local intensification of the heat exchange are conducted in the areas in which they are expected to occur.

Increase of the flow rate in third significantly extended turbulent zone of flow can be extremely beneficial when increase of the efficiency of the water transport system is required.

It can be applied in fire-fighting or to increase the efficiency of sewerage and anti-flood systems, or in transport of slurries, sludge and brines, when transported medium is going to be subjected to further filtration or other chemical processes. By the application of drag reduction effect pumping cost can be reduced significantly in such situations.

In case of anti-flood systems application biodegradable polymers should be used. High uncertainty regarding the toxicity is observed for cationic surfactants which are investigated in this paper. However, for such propose more toxic ionic surfactants can be replaced with nonionic, biodegradable surfactants for which drag reduction effect is also observed.

\section{References}

1. Y. Wang, B. Yu, J. L. Zakin, H. Shi, Advance in Mech. Eng., 10, 2011

2. J. L. Zakin, W. Ge, Polymer Physics: From Suspensions to Nanocomposites and Beyond, John Wiley \& Sons, Inc., Hoboken, NJ, USA, 89-127, 2010.

3. C. M. White, M. G. Mungal, Annu. Rev. Fluid Mech., 40, 235-256, 2008.

4. W. Borostow, 2008, J. Ind. Eng. Chem., 14, 409-416.

5. C. Shu-Peng, J. Hydrodynam., 24, 2, 202-206, 2010.

6. S. Tamano, M. Ito, K. Kato, K. Yokota, Phys. Fluids, 22, 5, 055102, 2010.

7. Z. Matras, Cracow University of Technology, Monografia 29, 1984.

8. T. Dujmovich, A. Gallegos, Offshore, $\mathbf{6 5}$, no. $12,1-4$, 2005.

9. J. F. Motier, L. C. Chou, N. S. Kommareddi, Proceedings of the ASME Fluids Engineering Division Summer Meeting, San Diego, Calif, USA, 1996.

10. R. C. R. Figueredo, E. Sabadini, Colloid. Surface. A, 215, no. 1-3, 77-86, 2003.

11. J. Golda, Chem. Eng. Commun., 43, no. 1-3, 53-67, 1986.

12. G. Dembek, H. W. Bewersdorff, $G W F$, Wasser/Abwasser, 122, no. 9, pp. 392-395, 1981.

13. N. A. Tuan, H. Mizunuma, J. Nonnewton. Fluid. Mech., 198, Pages 71-77, ISSN 0377-0257, 2013.
14. G. Aguilar, K. Gasljevic, E. F. Matthys, Int. J. Heat Mass Transfer., 44, 15, 2835-2843, 2001.

15. Z. Matras, T. Malcher, B. Gzyl-Malcher, Thin Solids Films, 516, 8848-8851, 2008.

16. Z. Matras, J. Głód, T. Malcher, Applied Mechanics and Engineering, 4, 441, 1999, 441-446, 1999.

17. B. Bębenek, Chem. and Proc. Engineering, 2, No 179, 1994.

18. A. Knop, R. Colby, ACS J. Surf. Colloids, 15, (1) 58$65,1999$.

19. J. Myska, AIChE Journal, 44, No 6., p.1467, 1998. 